\title{
Photon vs. proton radiochemotherapy: effects on brain tissue volume and perfusion
}

Jan Petr ${ }^{\mathrm{a}}$, Ivan Platzek ${ }^{\mathrm{b}}$, Frank Hofheinz ${ }^{\mathrm{a}}$, Henri J.M.M. Mutsaerts ${ }^{\mathrm{c}, \mathrm{d}, \mathrm{e}, \mathrm{f}, \mathrm{g}}$, Iris Asllani ${ }^{\mathrm{f}}$, Matthias J.P. van Osch ${ }^{\mathrm{h}}$, Annekatrin Seidlitz ${ }^{\mathrm{i}, \mathrm{j}, \mathrm{k}, \mathrm{l}}$, Pawel Krukowski ${ }^{\mathrm{m}}$, Andreas Gommlich ${ }^{\mathrm{j}, \mathrm{n}}$, Bettina Beuthien-Baumann ${ }^{\mathrm{l}, \mathrm{o}}$, Christina Jentsch ${ }^{\mathrm{i}, \mathrm{j}, \mathrm{l}, \mathrm{p}}$, Jens Maus ${ }^{\mathrm{a}}$, Esther G.C. Troost $\mathrm{t}^{\mathrm{i}, \mathrm{j}, \mathrm{k}, \mathrm{l}, \mathrm{n}, \mathrm{o}, \mathrm{p}}$, Michael Baumann $^{\mathrm{i}, \mathrm{j}, \mathrm{l,n}}$, Mechthild Krause ${ }^{* \mathrm{i}, \mathrm{j}, \mathrm{k}, \mathrm{l}, \mathrm{n}, \mathrm{p}}$, Jörg van den Hoff ${ }^{* a, o}$

\footnotetext{
${ }^{a}$ Helmholtz-Zentrum Dresden-Rossendorf, PET Center, Institute of Radiopharmaceutical Cancer Research, Dresden, Germany

${ }^{b}$ Department of Radiology, University Hospital Carl Gustav Carus, Technische Universität Dresden, Dresden, Germany

${ }^{c}$ Sunnybrook Research Institute, Toronto, Canada

${ }^{d}$ Department of Radiology, Academic Medical Center Amsterdam, the Netherlands

${ }^{e}$ Department of Radiology, University Medical Center Utrecht, the Netherlands

${ }^{f}$ Rochester Institute of Technology, Rochester, NY, USA

${ }^{g}$ Department of Radiology, VU University Medical Center, Amsterdam, the Netherlands

${ }^{h}$ Department of Radiology, Leiden University Medical Center, Leiden, Netherlands

${ }^{i}$ Department of Radiation Oncology, Faculty of Medicine and University Hospital Carl Gustav Carus, Technische Universität Dresden, Dresden, Germany

${ }^{j}$ OncoRay - National Center for Radiation Research in Oncology, Faculty of Medicine and University Hospital Carl Gustav Carus, Technische Universität Dresden, Germany

${ }^{k}$ German Cancer Consortium (DKTK) Dresden, Germany

${ }^{l}$ German Cancer Research Center (DKFZ) Heidelberg, Germany

${ }^{m}$ Department of Neuroradiology, University Hospital Carl Gustav Carus, Technische Universität Dresden, Dresden, Germany

${ }^{n}$ Helmholtz-Zentrum Dresden-Rossendorf, Institute of Radiooncology, Dresden, Germany

${ }^{\circ}$ Department of Nuclear Medicine, University Hospital Carl Gustav Carus, Technische Universität Dresden, Dresden, Germany

${ }^{p}$ NCT - National Center for Tumor Disease, Dresden, Germany
}

Title: 81 char. Text: 2990/3000 words. Pages: 20 .

Tables: 5. Figures: 1. Number of references: 38.

Corresponding author:

Jan Petr,

POB 5101 19, 01314 Dresden, Germany,

Phone +493512602709

j.petr@hzdr.de

\section{Running title (39/40 characters): Brain tissue changes after radiotherapy}

Keywords: arterial spin labeling, cerebral blood flow, radiochemotherapy, brain atrophy, proton beam therapy

*These authors contributed equally to the manuscript.

Email address: j.petr@hzdr.de (Jan Petr) 


\section{ABSTRACT}

Background and Purpose: To compare the structural and hemodynamic changes of healthy brain tissue in the cerebral hemisphere contralateral to the tumor following photon and proton radiochemotherapy.

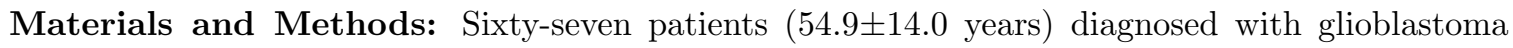
undergoing adjuvant photon $(n=47)$ or proton $(n=19)$ radiochemotherapy with temozolomide after tumor resection underwent T1-weighted and arterial spin labeling MRI. Changes in volume and perfusion before and 3 to 6 months after were compared between therapies.

Results: A decrease in gray matter $(\mathrm{GM})(-2.2 \%, P<0.001)$ and white matter (WM) $(-1.2 \%$, $P<0.001)$ volume was observed in photon-therapy patients compared to the pre-radiotherapy baseline. In contrast, for the proton-therapy group, no significant differences in GM $(0.3 \%, P=0.64)$ or WM $(-0.4 \%, P=0.58)$ volume were observed. GM volume decreased with $0.9 \%$ per 10 Gy dose increase $(P<0.001)$ and differed between the radiation modalities $(P<0.001)$. Perfusion decreased in photon-therapy patients $(-10.1 \%, P=0.002)$, whereas the decrease in proton-therapy patients, while comparable in magnitude, did not reach statistical significance $(-9.1 \%, P=0.12)$. There was no correlation between perfusion decrease and either dose $(P=0.64)$ or radiation modality $(P=0.94)$.

Conclusions: Our results show that the tissue volume decrease depends on radiation dose delivered to the healthy hemisphere and differs between treatment modalities. In contrast, the decrease in perfusion was comparable for both irradiation modalities. We conclude that proton therapy may reduce brain-volume loss when compared to photon therapy. 


\section{INTRODUCTION}

Glioblastoma is the most common primary malignant brain tumor in adults. The standard therapy is maximal surgical resection followed by radiotherapy (RT) with concurrent and adjuvant chemotherapy using temozolomide [1]. Both RT and chemotherapy are, however, associated with risks of cognitive deficits and structural and hemodynamic changes in the normal brain tissue $[2,3]$.

Brain atrophy may appear as a side-effect of RT and progressive decrease in gray matter (GM) volume over time and a dependence on radiation dose have been reported after photon RT $[4,5,6$, $7,8]$. Interestingly, no significant changes in white matter (WM) volume were observed in the same studies, despite findings WM fiber damage following RT from diffusion MRI data $[9,10]$.

RT was also shown to cause vessel-wall thickening and endothelial cell loss leading to cerebral microbleeds and occlusions in the microvasculature [11, 12]. These changes could in turn affect the healthy-tissue perfusion. Several studies have investigated the influence of RT on brain perfusion with mostly contradictory conclusions. Perfusion decreases have been detected by dynamic susceptibility contrast and arterial spin labeling (ASL) MRI, and with ${ }^{99 m}$ Tc-HMPAO scintigraphy $[13,14,15,16]$; whereas perfusion increases have been measured using a computed tomography (CT)-perfusion, dynamic susceptibility contrast MRI, and ${ }^{15} \mathrm{O}-\mathrm{H}_{2} \mathrm{O}$ PET $[17,18,19]$.

We posit that reduction of healthy tissue damage following radiochemotherapy (RCT) may be achieved by using proton instead of photon RT. Proton therapy offers better dose distribution whilst exploiting comparable biological effectiveness [20]. In this study, we aimed to assess potential benefits of proton over photon therapy in terms of reducing damage to healthy brain tissue. We investigated the early-delayed brain volume and perfusion changes in healthy tissue at 3 and 6 months after $\mathrm{RT}$, correlated the changes to the delivered radiation dose, and compared the differences between radiation modalities.

\section{METHODS}

\section{Participants and experimental design}

The present investigation concerns the first two follow-up sessions of patients treated in the prospective, two-arm (photon and proton therapy), single-center non-randomized imaging trial "Observational study of impact of $\left[{ }^{11} \mathrm{C}\right]$-methionine PET/MRI as a tool for individual tailoring postoperative radiochemotherapy for patients with glioblastoma multiforme" (PETra). This trial was designed to validate the value of $\left[{ }^{11} \mathrm{C}\right]$-methionine $\mathrm{PET}$ as an imaging biomarker for predicting the location of recurrence as a basis for future radiotherapy dose-escalation approaches. Patient accrual lasted from September 2013 until October 2016 and the results according to the endpoints laid down in the protocol will be reported separately.

The registered trial (NCT01873469) was conducted in accordance with the Declaration of Helsinki and was approved by the Ethics Committee of the Faculty of Medicine at the Technische Universität 
Dresden, Germany (EK41022013). All patients provided written informed consent.

The main inclusion criteria of the PETra trial included newly diagnosed glioblastoma, age $\geq 18$ years and Karnofsky Performance Score $\geq 60$. After clinical introduction of proton-beam therapy at the University Proton Therapy Dresden at December 2014, the choice of treatment with photon or proton-beam therapy depended on the decision of the treating physician, the patient and on reimbursement of the costs. The current investigation included only those patients for which MRI scans prior to initiation of RCT were available, who had unilateral tumor localization before RCT, who received all planned fractions of photon- or proton-beam irradiation, and who were scanned at least once after the end of RCT. In total, 72 patients (51 photon, 21 proton) were included in this imaging study (mean age $54.3 \pm 14.2$ years, range $23.2-81.8$ years, 29 female).

The first MR session was performed after surgery and before start of RCT. For each patient, imaging data also included $\left[{ }^{11} \mathrm{C}\right]$-Methionine PET and treatment-planning CT scans. RT treatment started $2-7$ weeks after full or partial tumor resection or biopsy. After the end of RT, follow-up MRI scans were acquired every 3 months for two years or until disease progression or drop out of the patient. Here we present follow-up data at 3 and 6 months after the end of RT.

Radiation treatment planning was based on the CT and PET/MRI scans. The margin of the clinical target volume (CTV) around the surgical cavity and macroscopic tumor was $20 \mathrm{~mm}$ for the volume treated up to 50 Gy (2 Gy per fraction) and $5 \mathrm{~mm}$ for the boost volume treated with an additional 10 Gy (2 Gy per fraction) to a total dose of 60 Gy (60 GyE for proton therapy). A 5mm margin was added for the planning target volume.

Photon-based radiation treatment plans were generated using either 3D conformal radiotherapy (3DCRT) (Oncentra Masterplan 3.1, Nucletron, Veenendaal, The Netherlands; n=27) or intensitymodulated radiotherapy (IMRT) (Pinnacle 9.0, Philips, Eindhoven, The Netherlands; n=24). Photon RT was delivered with linear accelerators with multileaf collimator (Siemens Healthcare, Erlangen, Germany) providing photons of energies 15 and 6 MV. Proton beam treatment plans were generated using passive double scattering technique (XiO, Nucletron) and therapy was delivered with a cyclotron providing energies of $100 \mathrm{MeV}-230 \mathrm{MeV}$.

Concomitant chemotherapy with the cytostatic agent temozolomide was performed according to Stupp et al. [1].

Image Acquisition

All imaging was performed on a 3T Philips Ingenuity TF PET/MRI scanner (Philips Healthcare, Best, The Netherlands) with an 8-channel head-coil.

On each session, a 3D Turbo Field Echo T1-weighted (T1w) image was acquired with a $1 \times$ $1 \times 1 \mathrm{~mm}^{3}$ resolution. A pseudo-continuous ASL sequence [21] with background suppression [22] was used to acquire perfusion-weighted images as described in detail previously [15]: voxel size $2.75 \times 2.75 \times 6 \mathrm{~mm}^{3}, 17$ slices (0.6 mm gap), 2D echo-planar-imaging readout, TR/TE $3765 / 11 \mathrm{~ms}, 30$ 
averages, labeling time $1650 \mathrm{~ms}$, post-labeling delay for the first and last slice 1525 and $2037 \mathrm{~ms}$, respectively. An $\mathrm{M}_{0}$ image was acquired with TR $5000 \mathrm{~ms}$.

Preprocessing and perfusion quantification

All image processing was fully automatic and was done using SPM12 (Wellcome Trust Centre for Neuroimaging, London, UK), and in-house routines written in Matlab (MathWorks, Natick, MA, USA) based on ExploreASL, with specific modifications to accommodate brain deformations by the tumor and surgery.

The T1w image was segmented using SPM12 [23] with enhanced tissue priors [24] providing a relative content of GM, WM, and CSF in each voxel, see Supplementary Figure 1B. To avoid the bias in segmentation caused by the presence of tumor and surrounding edema, both hemispheres were segmented separately. The T1w image was rigid-body co-registered with the mean ASL control image. Perfusion defined as regional cerebral blood flow (CBF) was quantified from the raw ASL data using the single compartment model [25] and provided voxel-wise in $\mathrm{mL} / \mathrm{min} / 100 \mathrm{~g}$, see perfusion maps in Supplementary Figure 1D.

For the pre-therapeutic sessions, the CT image with the radiation dose map was co-registered to the T1w image as shown in Supplementary Figure 1. For the post-therapeutic sessions, the $\mathrm{T} 1 \mathrm{w}$ images were non-linearly registered to the pre-therapeutic T1w images [26] to allow regional comparison of dose and volumes across sessions.

\section{Imaging data exclusion}

ASL images were examined by two researchers (JP, HM) with 7 years of experience in ASL image processing. Images with severe motion or acquisition artifacts were excluded from further analysis.

$\mathrm{T} 1 \mathrm{w}$ images were examined by a radiologist (IP) with 12 years of experience. Images with severe motion artifacts, which could lead to false decreases in GM volume [27], were excluded. Post-RCT sessions with new morphological findings in WM compared to the pre-RCT baseline (e.g. edema, leukoencephalopathy) were also excluded.

Post-RCT sessions with bilateral tumor progression, as assessed on contrast enhanced T1w images and PET images, were excluded from both perfusion and volume analyses.

\section{Statistical analysis}

Between-session changes in brain volume and perfusion were evaluated in the healthy hemisphere contralateral to the tumor. Volume of GM, WM, and total brain tissue (sum of GM and WM) in the hemisphere contralateral to the tumor was calculated automatically from the segmented $\mathrm{T} 1 \mathrm{w}$ images by summing the voxel-wise tissue content across all voxels. Mean GM perfusion was calculated as an average voxel-wise perfusion across the voxels with GM content exceeding $70 \%$. Relative volume and perfusion changes between sessions $\mathrm{A}$ and $\mathrm{B}$ were calculated as $100 \% *$ mean value $_{B}-$

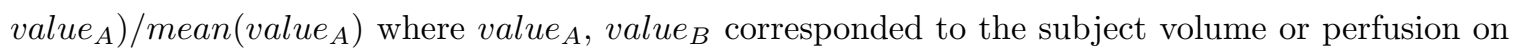


sessions A, B, respectively. A two-tailed paired t-test was used to assess the statistical significance of the differences. Normality of the distribution was verified by a Shapiro-Wilk test prior to application of the t-test. $P<0.05$ was considered statistically significant in all analyses. Differences were compared between the photon- and proton-therapy patient groups and also for the two subgroups of the photon therapy - 3DCRT and IMRT.

To study radiation-dose dependence, dose regions were created for each participant by categorizing the voxels of the healthy hemisphere according to received dose into bins of $0-10,10-60,10-20$, 20-30, 30-60 Gy. Both narrower bins and no division of regions with dose above 10 Gy were used to be able to study high-dose regions in more detail and also to accommodate for the fact that the amount of voxels in high-dose regions was small for the proton therapy. Statistical significance of the differences from a zero change and a change compared to the region with dose below 10 Gy was tested using simple and paired t-tests, respectively. For the perfusion analysis, dose-regions were restricted to voxels with $\mathrm{GM} \geq 70 \%$ and regions smaller than $5 \mathrm{~cm}^{3}$ were excluded. For the volume analysis, a threshold of $35 \mathrm{~cm}^{3}$ was used since this analysis was not restricted to GM voxels. Both thresholds were approximately $5 \%$ of the total considered volume of around $150 \mathrm{~cm}^{3}$ (for perfusion) and $700 \mathrm{~cm}^{3}$ (for volume).

Additionally, a multivariate linear regression was performed to investigate to what extent the total tissue volume or perfusion was influenced by patient age, time from the baseline scan, and mean dose received in the healthy hemisphere. The radiation modality was not included in the multivariate analysis as it was strongly correlated with the mean dose $(R=-0.80, P=1.2 \mathrm{e}-22)$.

\section{RESULTS}

Patient characteristics

After excluding images with artifacts, 41 photon- (22 3DCRT and 19 IMRT) and 16 protontherapy patients were included in the volume analysis, and 44 photon- (25 3DCRT and 19 IMRT) and 16 proton-therapy patients were included in the perfusion analysis, see Table 1. Details of exclusion reasons are provided in Supplementary Tables 1 and 2. There was no difference between the protonand photon-therapy groups and subgroups in neither of age $(\mathrm{P}>0.25)$, ECOG score $(\mathrm{P}>0.11)$, BMI $(\mathrm{P}>0.23)$, or chemotherapy duration $(\mathrm{P}>0.34)$ for neither of the volume and perfusion analysis. CTV did not differ between proton and photon-therapy groups ( $\mathrm{P}>0.73)$, but there was around $20 \%$ difference between the two photon-therapy subgroups $(\mathrm{P}<0.022)$. Example of mean dose distribution and dose histograms for the different RT modalities are shown in Supplementary Figure 3 and 4, respectively. The average mean dose in the healthy hemisphere was 19.1 Gy for the CRT-, 26.2 Gy for the IMRT-, 22.2 Gy for the photon-, and 4.2 Gy for the proton-therapy group and the difference was significant between all groups. 


\section{Tissue volume changes following RT treatment}

For all photon-therapy patients on both follow-up sessions, both GM and WM volumes decreased compared to the baseline (between -1.2 and $-3.0 \%, P<0.003$, Table 2, Figure 1) whereas no significant changes were observed for the proton-therapy patients (between 0.3 and $-0.8 \%, P>$ 0.27 , Table 2). The effect of RT modality on volume changes was significant $\left(P=3 \cdot 10^{-4}\right)$. The parenchymal volume seems to further decrease between the two post-therapeutic sessions $(-0.8 \%$, $\mathrm{P}>0.027$ for the photon-; and $-0.8 \%, \mathrm{P}>0.0025$ for the proton-therapy group). The volume decreased significantly for both the 3DCRT and IMRT subgroups with higher decrease in the IMRT group. On the first post-therapy session, the volume decrease was significantly higher in both the 3DCRT $(\mathrm{P}=0.045)$ and the IMRT $(\mathrm{P}=0.0049)$ groups than in the proton-therapy group, however, was not different between the 3DCRT and IMRT groups $(\mathrm{P}=0.15)$.

Dose-dependent GM volume changes are provided in Table 3. The GM volume decreased significantly on the first post-therapeutic session compared to the pre-therapeutic session in all regions for the photon-therapy patients (up to $-3.1 \%, P=10^{-5}$ ). In the regions with dose above $10 \mathrm{~Gy}$, the decrease was almost two times as high as in the region with dose below 10 Gy $(-1.6 \%, P=0.026)$. Significant decreases in GM volume on the second post-therapeutic session compared to the pretherapeutic session were also observed in all patients (between $-1.6 \%$ and $-3.6 \%, P<0.018$ ) except in the region with dose below 10 Gy $(-1.6 \%, P=0.12)$. Significant differences $(P<0.032)$ between the volume decrease in high-dose $(-3.2--4.1 \%)$ than in the low-dose $(0.6--0.4 \%)$ regions were observed in the proton-therapy as well despite the small size of the high-dose regions.

The results of the multivariate linear regression showed that the mean received dose in the healthy hemisphere, time after treatment and age had a statistically significant effect on the volume decrease of $0.9 \% / 10 \mathrm{~Gy}\left(P=10^{-5}\right), 0.25 \% /$ month $(P=0.026)$, and $0.03 \%(P=0.021)$, respectively, while the effect of $\operatorname{sex}(\beta=-0.59, P=0.13)$, BMI $(\beta=0.07, P=0.14)$, chemotherapy duration $(\beta=-0.02$, $P=0.63)$, and $\operatorname{CTV}(\beta=0.00, P=0.74)$ was not statistically significant. Scatter plots of tissue-volume decrease vs. mean dose, time, and age, respectively, are shown in Supplementary Figure 2.

\section{Gray matter perfusion changes following RT treatment}

The mean relative perfusion changes in the whole healthy hemisphere are shown in Table 4. Statistically significant decrease in perfusion was observed on both post-therapeutic sessions compared to the pre-therapeutic session for the photon-therapy patients (relative change was $-10.1 \%, P=0.002$ on first; and $-11.1 \%, P=0.008$ on the second post-therapeutic session). The magnitude of the effect was similar for the proton-therapy patients $(-9.1 \%, P=0.12$ on first; and $-8.8 \%, P=0.17$ on the second post-therapeutic session). Although the perfusion decrease in proton-therapy patients did not reach statistical significance there was no effect size difference between the photon- and proton-therapy results $(P=0.94)$. No significant change was observed between the two post-therapeutic sessions 
$(\mathrm{P}>0.5)$. Although the perfusion decrease was higher for the IMRT than for the 3DCRT subgroup, the difference was not significant on neither of the follow-up sessions $(\mathrm{P}>0.47)$.

The perfusion changes for different dose regions are shown in Table 5. For the photon-therapy patients, significant perfusion decreases were observed for all doses above 10 Gy for the first posttherapeutic session compared to the pre-therapeutic session. However, there were no significant differences in perfusion decrease between regions below and above $10 \mathrm{~Gy}$. In line with the observed global perfusion decrease, perfusion decrease in proton-therapy patients was comparable in magnitude, albeit not statistically significant.

The multivariate linear regression did not reveal significant effects of age $(\beta=-0.24, P=0.15)$, mean dose $(\beta=0.10, P=0.64)$, time after therapy $(\beta=-0.32, P=0.8)$, sex $(\beta=-4.14, P=0.35)$, BMI $(\beta=-0.19, P=0.73)$, chemotherapy duration $(\beta=0.50, P=0.30)$, or $\operatorname{CTV}(\beta=0, P=1.0)$ on wholehemisphere perfusion changes.

\section{DISCUSSION}

We compared the early-delayed effects of photon versus proton radiotherapy in the healthy brain tissue of glioblastoma patients. Our three main findings are: (1) There was less brain-tissue volume loss in patients who underwent proton-therapy compared to those who underwent photon-therapy; (2) Loss of brain-tissue volume strongly correlated with the mean radiation dose received in the healthy hemisphere; (3) Perfusion decreased in all patients, independent of therapy modality or radiation dose. The effect size of the perfusion decrease following therapy was similar between the two groups but reached statistical significance only for the photon-therapy patients. These findings are in agreement with the hypothesis that proton therapy reduces the volume loss of healthy tissue compared to photon therapy simply through limiting the radiation dose received in the contralateral hemisphere.

The observation of brain-tissue loss in photon-therapy but not in proton-therapy patients, in combination with the dose-dependency of the tissue loss suggests that brain volume changes are caused mainly by the radiation effects. Normal aging is estimated to cause between $0.26 \%$ and $0.39 \%$ per year decrease in GM volume, which is less than the values observed in the current study [28, 29]. A dose dependent GM-volume decrease was previously described by Karunamuni et al.[5]. Our results are in agreement with their reported effect size (1 - 5\% GM-volume decrease). However, the results are not directly comparable as the probability distribution of volume changes and not the mean volume changes were reported by Karunamuni et al.[5]. An approximately 5\% GM-volume decrease reported for the same tumor type and therapy by Prust et al.[4] in 8 patients is close to our finding of $2-3 \%$ GM-volume decrease. The remaining difference could be caused by the fact that Prust et al.[4] performed the T1w image segmentation in the whole brain at once without excluding sessions with bilateral tumors and edemas in the contralateral hemisphere. The caveat is 
that the SPM12 segmentation employs a global model assuming homogeneous GM and WM intensity distribution across the brain on the T1w images. Longitudinal intensity changes in WM around the tumor (e.g. appearance of edema in follow-up measurement) will thus influence the segmentation results also in the contralateral hemisphere and therefore can cause false classification of part of GM tissue as WM, leading to false interpretation as a GM-volume decrease accompanied by a WMvolume increase as noted by Chamberlain et al.[30]. False volume variations due to the mentioned sources of error might explain why a WM-volume decrease has not been reported previously. We have circumvented this issue by segmenting the healthy hemisphere separately and by also investigating volume changes of GM and WM tissue combined.

In this study, approximately $10 \%$ perfusion decrease was present in both irradiation modalities irrespective of the received radiation dose. The decrease was statistically significant for photon therapy but not for proton therapy. Since the effect size and variability were similar for both therapies the difference in statistical significance can be understood as a consequence of the smaller sample size of the proton-therapy group.

Tumor perfusion measured in glioblastoma patients using MRI with or without contrast can be used before or following RCT to detect residual or recurrent tumor, pseudo-progression or to predict time to progression [31, 32, 33]. In such instances, tumor perfusion is often normalized to perfusion in contralateral normal-appearing brain tissue. Therefore, our finding of perfusion decreases in the normal-appearing brain tissue receiving relatively low radiation dose is a potential confounder for the normalized perfusion and should be taken into consideration. While vascular damage and neurocognitive deficits typically occur several years after RCT, we observed a perfusion decrease three to six months after the therapy. In several recent studies, perfusion decrease has been proposed as an early predictor of cognitive decline in neurodegenerative disease [34, 35] and similar effects might be present following photon- or proton-based RCT. This, however, requires validation by longer follow-up coupled with neurocognitive testing.

Previous studies that reported on perfusion changes following RCT were mostly conducted in small populations and differed in received radiation dose, irradiation region definition, time after RT, and imaging modality used. This may explain why previously reported results contradict one another. Price et al.[14] observed a perfusion decrease only in the regions and above 32 Gy, Taki et al. [13] reported $7 \%$ perfusion at 3 months after radiosurgery in a region that received less than 5 Gy, and Hahn et al.[18] observed a CBF increase using $\left[{ }^{15} \mathrm{O}\right] \mathrm{H}_{2} \mathrm{O}$ PET measurements. However, these studies were conducted with 6 patients or less. Directly comparable to the current study in terms of patient group characteristics, treatment type and statistical analysis is one investigation using dynamic susceptibility contrast MRI [16] and our previous investigation using ASL [15]. Both show similar results of around 10\% GM CBF decrease in 10 and 24 patients, respectively. A small increase in CBF with increased dose has been observed in all three of these studies without, however, reaching 
statistical significance. The changes in perfusion thus do not appear to be mainly caused by RT, but seem related to either surgery, chemotherapy, or the disease itself. Especially the ongoing monthly chemotherapy after the end of RT can cause further damage by the synergistic effect of chemo and radiation therapy, as higher concentrations of chemotherapeutic agents in normal brain tissue is achieved due to increased leakage through the bloodbrain barrier caused by RT [36]. The activity of $\mathrm{O}^{6}$-Methylguanine-DNA methyltransferase (MGMT) has been related to both better therapeutic response of glioblastoma and normal tissue protection to radiochemotherapy with $\mathrm{O}^{6}$-alkylating agents such as temozolomide [37, 38]. While increase of MGMT activity could in theory play role in the decrease of the perfusion and structural damage, we were not able to study this due to lack of MGMT data in the normal tissue. We have observed higher, albeit mostly not significant, perfusion and volume decreases in the IMRT group versus the 3DCRT group. For the volume changes, this can be partly explained by the higher mean dose to the contralateral hemisphere in the IMRT group. Only minimal correlation with mean dose, however, was found for the perfusion decrease. The reason for the differences in the perfusion changes can thus be either a larger relative volume irradiated to high radiation doses of i 30 Gy or by another, unknown effect. There was a significant difference in the CTV between the 3DCRT and the IMRT groups. It thus remains difficult to assess to what extent the difference in radiation dose was caused by the varying CTV or the radiation treatment technique. Although the CTV difference was not large, further studies are needed to provide an unbiased comparison of the effects of 3DCRT and IMRT on the healthy hemisphere.

Our findings indicate that early-delayed brain-tissue volume changes are strongly dependent on the radiation dose applied to the healthy brain, suggesting that a reduced dose to brain-tissue may reduce regional loss of brain volume. Indeed, proton-therapy patients were shown to have significantly lower loss of brain tissue than the photon-therapy patients. On the other hand, perfusion decreased irrespective of the beam modality. Both longer follow-up in a larger cohort, as well as neurocognitive testing may allow to further explore the underlying pathological pathways of primary and secondary damage and its effect on cognitive function and to investigate if these benefits of proton therapy prevail over longer time.

\section{Conflict of Interest Statement}

The authors have nothing to disclose.

\section{REFERENCES}

[1] Stupp R, Mason WP, van den Bent MJ, Weller M, Fisher B, Taphoorn MJB, Belanger K, Brandes AA, Marosi C, Bogdahn U, Curschmann J, Janzer RC, Ludwin SK, Gorlia T, Allgeier A, Lacombe D, Cairncross JG, Eisenhauer E, Mirimanoff RO. Radiotherapy plus con- 
comitant and adjuvant temozolomide for glioblastoma. The New England journal of medicine. 2005;352(10):987-996.

[2] Armstrong CL, Gyato K, Awadalla AW, Lustig R, Tochner ZA. A Critical Review of the Clinical Effects of Therapeutic Irradiation Damage to the Brain: The Roots of Controversy. Neuropsychology Review. 2004;14(1):65-86.

[3] Ahles TA, Root JC, Ryan EL. Cancer- and cancer treatment-associated cognitive change: An update on the state of the science. Journal of Clinical Oncology. 2012;30(30):3675-3686.

[4] Prust MJ, Jafari-Khouzani K, Kalpathy-Cramer J, Polaskova P, Batchelor TT, Gerstner ER, Dietrich J. Standard chemoradiation for glioblastoma results in progressive brain volume loss. Neurology. 2015;85(8):683-691.

[5] Karunamuni RA, Moore KL, Seibert TM, Li N, White NS, Bartsch H, Carmona R, Marshall D, McDonald CR, Farid N, Krishnan A, Kuperman J, Mell LK, Brewer J, Dale AM, Moiseenko V, Hattangadi-Gluth JA. Radiation sparing of cerebral cortex in brain tumor patients using quantitative neuroimaging. Radiotherapy and Oncology. 2016;118(1):29-34.

[6] Karunamuni R, Bartsch H, White NS, Moiseenko V, Carmona R, Marshall DC, Seibert TM, McDonald CR, Farid N, Krishnan A, et al. Dose-dependent cortical thinning after partial brain irradiation in high-grade glioma. International Journal of Radiation Oncology* Biology* Physics. 2016;94(2):297-304.

[7] Seibert TM, Karunamuni R, Bartsch H, Kaifi S, Krishnan AP, Dalia Y, Burkeen J, Murzin V, Moiseenko V, Kuperman J, White NS, Brewer JB, Farid N, McDonald CR, Hattangadi-Gluth JA. Radiation Dose-Dependent Hippocampal Atrophy Detected With Longitudinal Volumetric Magnetic Resonance Imaging. International Journal of Radiation Oncology*Biology*Physics. 2017;97(2):263-269.

[8] Seibert TM, Karunamuni R, Kaifi S, Burkeen J, Connor M, Krishnan AP, White NS, Farid N, Bartsch H, Murzin V, et al. Cerebral cortex regions selectively vulnerable to radiation dose-dependent atrophy. International Journal of Radiation Oncology* Biology* Physics. 2017;97(5):910-918.

[9] Chapman CH, Zhu T, Nazem-Zadeh M, Tao Y, Buchtel HA, Tsien CI, Lawrence TS, Cao Y. Diffusion tensor imaging predicts cognitive function change following partial brain radiotherapy for low-grade and benign tumors. Radiotherapy and Oncology. 2016;120(2):234-240.

[10] Connor M, Karunamuni R, McDonald C, White N, Pettersson N, Moiseenko V, Seibert T, Marshall D, Cervino L, Bartsch H, et al. Dose-dependent white matter damage after brain radiotherapy. Radiotherapy and Oncology. 2016;121(2):209-216. 
[11] OConnor MM, Mayberg MR. Effects of radiation on cerebral vasculature: a review. Neurosurgery. 2000;46(1):138-151.

[12] Omuro AMP, Ben-Porat LS, Panageas KS, Kim AK, Correa DD, Yahalom J, Deangelis LM, Abrey LE. Delayed neurotoxicity in primary central nervous system lymphoma. Archives of neurology. 2005;62(10):1595-1600.

[13] Taki S, Higashi K, Oguchi M, Tamamura H, Tsuji S, Ohta K, Tonami H, Yamamoto I, Okamoto $\mathrm{K}$, Iizuka H. Changes in regional cerebral blood flow in irradiated regions and normal brain after stereotactic radiosurgery. Annals of nuclear medicine. 2002;16(4):273-277.

[14] Price SJ, Jena R, Green HaL, Kirkby NF, Lynch AG, Coles CE, Pickard JD, Gillard JH, Burnet NG. Early radiotherapy dose response and lack of hypersensitivity effect in normal brain tissue: a sequential dynamic susceptibility imaging study of cerebral perfusion. Clinical oncology. 2007;19(8):577-587.

[15] Petr J, Platzek I, Seidlitz A, Mutsaerts HJMM, Hofheinz F, Schramm G, Maus J, BeuthienBaumann B, Krause M, van den Hoff J. Early and late effects of radiochemotherapy on cerebral blood flow in glioblastoma patients measured with non-invasive perfusion MRI. Radiotherapy and Oncology. 2016;118(1):24-28.

[16] Fahlström M, Nyholm T, Larsson EM, Are radiation-induced perfusion changes in normal appearing brain tissue a confounding factor in tumour response evaluation with DSC-MRI? In: 25th Annual meeting of the International Society for Magnetic Resonance in Medicine, . 2017. p 2896.

[17] Millar BAM, Purdie TG, Yeung I, Pond GR, Billingsley S, Wong R, Haddad P, Wong CS, Laperriere N. Assessing perfusion changes during whole brain irradiation for patients with cerebral metastases. Journal of Neuro-Oncology. 2005;71(3):281-286.

[18] Hahn Ca, Zhou SM, Raynor R, Tisch A, Light K, Shafman T, Wong T, Kirkpatrick J, Turkington T, Hollis D, Marks LB. Dose-dependent effects of radiation therapy on cerebral blood flow, metabolism, and neurocognitive dysfunction. International journal of radiation oncology, biology, physics. 2009;73(4):1082-1087.

[19] Jakubovic R, Sahgal A, Ruschin M, Pejovic-Milic A, Milwid R, Aviv RI. Non Tumor Perfusion Changes Following Stereotactic Radiosurgery to Brain Metastases. Technol Cancer Res Treat. 2015;14(4):497-503.

[20] Levin WP, Kooy H, Loeffler JS, DeLaney TF. Proton beam therapy. Br J Cancer. 2005;93(8):849-854. 
[21] Dai W, Garcia D, de Bazelaire C, Alsop DC. Continuous flow-driven inversion for arterial spin labeling using pulsed radio frequency and gradient fields. Magn Reson Med. 2008;60(6):14881497.

[22] Garcia DM, Duhamel G, Alsop DC. Efficiency of inversion pulses for background suppressed arterial spin labeling. Magn Reson Med. 2005;54(2):366-372.

[23] Ashburner J, Friston KJ. Unified segmentation. Neuroimage. 2005;26(3):839-851.

[24] Lorio S, Fresard S, Adaszewski S, Kherif F, Chowdhury R, Frackowiak R, Ashburner J, Helms G, Weiskopf N, Lutti A, Draganski B. New tissue priors for improved automated classification of subcortical brain structures on MRI. Neuroimage. 2016;130:157-166.

[25] Alsop DC, Detre Ja, Golay X, Günther M, Hendrikse J, Hernandez-Garcia L, Lu H, MacIntosh BJ, Parkes LM, Smits M, van Osch MJP, Wang DJJ, Wong EC, Zaharchuk G. Recommended implementation of arterial spin-labeled perfusion MRI for clinical applications: A consensus of the ISMRM perfusion study group and the European consortium for ASL in dementia. Magn Reson Med. 2015;73(1):102-116.

[26] Ashburner J, Ridgway G. Symmetric diffeomorphic modeling of longitudinal structural mri. Frontiers in Neuroscience. 2013;6:197.

[27] Reuter M, Tisdall MD, Qureshi A, Buckner RL, van der Kouwe AJW, Fischl B. Head motion during MRI acquisition reduces gray matter volume and thickness estimates. NeuroImage. 2015;107:107-115.

[28] Good CD, Johnsrude IS, Ashburner J, Henson RNA, Friston KJ, Frackowiak RSJ. A VoxelBased Morphometric Study of Ageing in 465 Normal Adult Human Brains. NeuroImage. 2001;14(1):21-36.

[29] Scahill R, Frost C, Jenkins R, Whitwell J, Rossor M, Fox N. A longitudinal study of brain volume changes in normal aging using serial registered magnetic resonance imaging. Archives of Neurology. 2003;60(7):989-994.

[30] Chamberlain MC, Dietrich J, Prust M. Standard chemoradiation for glioblastoma results in progressive brain volume loss: Author Response. Neurology. 2016;86(10):979-979.

[31] Lindner T, Ahmeti H, Lübbing I, Helle M, Jansen O, Synowitz M, Ulmer S. Intraoperative resection control using arterial spin labeling - proof of concept, reproducibility of data and initial results. NeuroImage: Clinical. 2017;15:136-142. 
[32] Nael K, Bauer AH, Hormigo A, Lemole M, Germano IM, Puig J, Stea B. Multiparametric mri for differentiation of radiation necrosis from recurrent tumor in patients with treated glioblastoma. American Journal of Roentgenology. 2017;p 1-6.

[33] Park JE, Ryu KH, Kim HS, Kim HW, Shim WH, Jung SC, Choi CG, Kim SJ, Kim JH. Perfusion of surgical cavity wall enhancement in early post-treatment mr imaging may stratify the time-to-progression in glioblastoma. PLOS ONE. 2017;12(7):1-13.

[34] Steketee RME, Bron EE, Meijboom R, Houston GC, Klein S, Mutsaerts HJMM, Mendez Orellana CP, de Jong FJ, van Swieten JC, van der Lugt A, Smits M. Early-stage differentiation between presenile Alzheimer's disease and frontotemporal dementia using arterial spin labeling MRI. European Radiology. 2016;26(1):244-253.

[35] Wang Z. Characterizing early Alzheimer's disease and disease progression using hippocampal volume and arterial spin labeling perfusion MRI. J Alzheimers Dis. 2014;42 Suppl 4:S495-502.

[36] Taphoorn MJB, Klein M. Cognitive deficits in adult patients with brain tumours. The Lancet Neurology. 2004;3(3):159-168.

[37] Christmann M, Verbeek B, Roos WP, Kaina B. O6-Methylguanine-DNA methyltransferase (MGMT) in normal tissues and tumors: Enzyme activity, promoter methylation and immunohistochemistry. Biochimica et Biophysica Acta - Reviews on Cancer. 2011;1816(2):179-190.

[38] Kaina B, Margison GP, Christmann M. Targeting O 6-methylguanine-DNA methyltransferase with specific inhibitors as a strategy in cancer therapy. Cellular and Molecular Life Sciences. 2010;67(21):3663-3681. 


\section{TABLES}

Table 1: Patients' demographics.

\begin{tabular}{lcccc}
\hline & \multicolumn{2}{c}{ Volume analysis } & \multicolumn{2}{c}{ Perfusion analysis } \\
& Photon & Proton & Photon & Proton \\
\hline Patients [n] & 41 & 16 & 44 & 16 \\
Male/female [n] & $24 / 17$ & $10 / 6$ & $24 / 20$ & $11 / 5$ \\
Mean age [years] & $55.9 \pm 13.9$ & $51.3 \pm 16.3$ & $55.4 \pm 13.3$ & $51.5 \pm 16.2$ \\
Age range [years] & $25.3-81.8$ & $23.2-72.4$ & $25.3-75.3$ & $23.2-72.4$ \\
\hline ECOG Score & $0.5 \pm 0.6$ & $0.4 \pm 0.5$ & $0.6 \pm 0.6$ & $0.5 \pm 0.6$ \\
BMI [kg/m ${ }^{2}$ ] & $26.2 \pm 4.8$ & $24.9 \pm 3.9$ & $26.3 \pm 4.6$ & $25.9 \pm 3.8$ \\
Tumor hemisphere [L/R] & $19 / 22$ & $10 / 6$ & $21 / 23$ & $11 / 5$ \\
Tumor location & & & & \\
Frontal & 21 & 5 & 22 & 7 \\
Temporal & 9 & 7 & 6 & 6 \\
Parietal & 3 & 2 & 5 & 1 \\
Occipital & 2 & 0 & 3 & 0 \\
Frontotemporal & 0 & 1 & 0 & 1 \\
Temporooccipital & 3 & 1 & 4 & 1 \\
Parietooccipital & 1 & 0 & 1 & 0 \\
Thalamus & 2 & 0 & 2 & 0 \\
Cerebellum & 0 & 0 & 1 & 0 \\
Biopsy only & 2 & 0 & 2 & 0 \\
\hline CTV [mm ${ }^{3}$ ] & $224.8 \pm 224.8$ & $217.4 \pm 217.4$ & $226.8 \pm 226.8$ & $229.4 \pm 229.4$ \\
Mean dose [Gy] & $22.6 \pm 6.6$ & $4.0 \pm 4.4$ & $22.4 \pm 7.1$ & $4.9 \pm 4.4$ \\
Simultaneous TMZ [days] & $40.2 \pm 4.8$ & $38.4 \pm 7.5$ & $40.5 \pm 4.6$ & $39.8 \pm 5.6$ \\
Dexamethasone [n (\%)] & $4(10 \%)$ & $1(6 \%)$ & $5(11 \%)$ & $1(6 \%)$ \\
\hline Par & & & & \\
\end{tabular}

Patients' demographics of the groups of photon and proton-therapy patients used for the volume and perfusion analysis. Additionally, the clinical target volume, the mean radiation dose in the healthy hemisphere, the number of days during which $75 \mathrm{mg} / \mathrm{m}^{2} /$ day of Temozolomide was administered simultaneously to the RT treatment, and the number of patients receiving Dexamethasone in time between the baseline and second follow-up measurement are given. The difference between the two photon-therapy groups and between the proton-therapy group was not significant $(\mathrm{P}>0.05)$ for neither of the parameters (Age, ECOG, BMI and TMZ) for neither of the analysis (volume, perfusion). CTV was different between the two photon-therapy subgroups $(\mathrm{P}<0.022)$ but not between proton and photon-therapy groups $(\mathrm{P}>0.73)$. Mean dose was different both between the two photon-therapy subgroups $(\mathrm{P}<0.002)$ and between the proton- and photon-therapy groups $\left(\mathrm{P}<10^{-8}\right)$. 
Table 2: Global brain volume changes in the healthy hemisphere.

\begin{tabular}{ccccccccccc}
\hline $\begin{array}{c}\text { RT } \\
\text { modal. }\end{array}$ & \multicolumn{2}{c}{ Session } & num. & B & interval & \multicolumn{2}{c}{$\Delta \mathrm{GM}$} & \multicolumn{2}{c}{$\Delta$ WM } & \multicolumn{2}{c}{$\Delta$ Tissue } \\
\multirow{2}{*}{ Photon } & Pre & Post-1 & 40 & $4.7 \pm 0.4$ & $-2.2 \pm 3.0$ & $6 \cdot 10^{-5}$ & $-1.2 \pm 1.9$ & 0.0004 & $-1.7 \pm 1.7$ & $10^{-7}$ \\
& Pre & Post-2 & 24 & $7.9 \pm 0.5$ & $-2.2 \pm 3.2$ & 0.003 & $-3.0 \pm 2.6$ & $10^{-5}$ & $-2.5 \pm 2.0$ & $2 \cdot 10^{-6}$ \\
\hline \multirow{2}{*}{ Proton } & Pre & Post-1 & 15 & $4.7 \pm 0.4$ & $0.3 \pm 2.3$ & 0.64 & $-0.4 \pm 3.0$ & 0.58 & $-0.0 \pm 1.9$ & 0.98 \\
& Pre & Post-2 & 12 & $8.0 \pm 0.7$ & $-0.8 \pm 2.8$ & 0.36 & $-1.0 \pm 3.0$ & 0.27 & $-0.9 \pm 2.1$ & 0.2 \\
\hline \hline \multirow{2}{*}{3 DCRT } & Pre & Post-1 & 21 & $4.7 \pm 0.4$ & $-1.4 \pm 2.8$ & 0.035 & $-1.4 \pm 1.9$ & 0.003 & $-1.4 \pm 1.6$ & 0.001 \\
& Pre & Post-2 & 13 & $7.9 \pm 0.5$ & $-1.1 \pm 3.4$ & 0.29 & $-2.7 \pm 3.2$ & 0.01 & $-1.8 \pm 2.1$ & 0.012 \\
\hline \multirow{2}{*}{ IMRT } & Pre & Post-1 & 19 & $4.7 \pm 0.4$ & $-3.0 \pm 3.0$ & 0.0005 & $-0.9 \pm 2.0$ & 0.058 & $-2.1 \pm 1.7$ & $4 \cdot 10^{-5}$ \\
& Pre & Post-2 & 11 & $7.9 \pm 0.5$ & $-3.6 \pm 2.3$ & 0.0005 & $-3.5 \pm 1.8$ & $5 \cdot 10^{-5}$ & $-3.5 \pm 1.3$ & $4 \cdot 10^{-6}$ \\
\hline
\end{tabular}

Tissue volume changes for the first (Post-1) and second (Post-2) post-radiotherapy sessions are shown compared to the pre-therapy baseline (Pre). Significant mean relative volume decreases in GM, WM, and brain tissue were observed in the photon-therapy patients. Volume changes in the proton-therapy patients were not significant and the magnitudes of the changes were also much smaller than in the photon-therapy patients. Results are shown also for the two subgroups of the photon-therapy patients. Volume decreased in both subgroups on both sessions and the effect was stronger in the IMRT than in the 3DCRT patients. 
Table 3: Dose-dependent brain volume changes in the healthy hemisphere.

\begin{tabular}{ccccccc}
\hline Photon & Dose [Gy] & $0-10$ & $10-60$ & $10-20$ & $20-30$ & $30-60$ \\
\hline Pre & $\Delta$ GM [\%] & -1.6 & -2.4 & -1.8 & -2.9 & -3.1 \\
$/$ & Region size [\%/n] & $23 \% / 32$ & $77 \% / 40$ & $27 \% / 39$ & $21 \% / 34$ & $29 \% / 37$ \\
Post-1 & P-value [0] & $0.026^{\star}$ & $10^{-5 \star \star}$ & $0.0004^{\star \star}$ & $3 \cdot 10^{-6 \star \star}$ & $10^{-5 \star \star}$ \\
& P-value [LD] & - & $0.027^{\star}$ & $0.032^{\star}$ & 0.067 & $0.014^{\star}$ \\
\hline Pre & $\Delta$ GM [\%] & -1.6 & -2.5 & -1.8 & -2.5 & -3.6 \\
$/$ & Region size [\%/n] & $21 \% / 17$ & $79 \% / 24$ & $29 \% / 23$ & $20 \% / 20$ & $29 \% / 22$ \\
Post-2 & P-value [0] & 0.12 & $0.0008^{\star \star}$ & $0.018^{\star}$ & $0.004^{\star}$ & $6 \cdot 10^{-5 \star \star}$ \\
& P-value [LD] & - & 0.31 & 0.78 & 0.45 & 0.13 \\
\hline Proton & Dose [Gy] & $0-10$ & $10-60$ & $10-20$ & $20-30$ & $30-60$ \\
\hline Pre & $\Delta$ GM [\%] & 0.6 & -3.2 & - & - & - \\
$/$ & Region size [\%/n] & $91 \% / 15$ & $9 \% / 6$ & $2 \% / 0$ & $2 \% / 0$ & $5 \% / 6$ \\
Post-1 & P-value [0] & 0.3 & 0.14 & - & - & - \\
& P-value [LD] & - & $0.0063^{\star}$ & - & - & - \\
\hline Pre & $\Delta$ GM [\%] & -0.4 & -4.1 & - & - & - \\
$/$ & Region size [\%/n] & $90 \% / 12$ & $10 \% / 7$ & $2 \% / 0$ & $2 \% / 0$ & $6 \% / 7$ \\
Post-2 & P-value [0] & 0.57 & 0.082 & - & - & - \\
& P-value [LD] & - & $0.032^{\star}$ & - & - & - \\
\hline
\end{tabular}

The mean relative GM volume changes are shown for several dose regions. Note that the effect size and $P$-values are not given for regions (mainly proton therapy) with relative size under $7 \%$ as they were considered too much affected by noise. For each session, the mean dose-region volume as a percentage of the whole-hemisphere volume, and the number of regions that exceeded the $35 \mathrm{~cm}^{3}$ threshold are given. The $P$-values of the t-tests for probability of no absolute change [0] and no change relative to the low dose (below $10 \mathrm{~Gy}$ ) region [LD] are shown. Statistically significant values are labeled by ${ }^{\star}$ for $P<0.05$ and by ${ }^{\star \star}$ for $P<0.001$. For the photon-therapy patients, a significant decrease of GM volume was observed for all doses except for the region with dose below $10 \mathrm{~Gy}$ on the second post-therapeutic session. On the first post-therapeutic session, significantly higher GM volume decrease was observed in the regions above 10 Gy than in the region below $10 \mathrm{~Gy}$. None of the proton-therapy results were significant. 
Table 4: Global perfusion changes in the healthy hemisphere.

\begin{tabular}{ccccccccc}
\hline $\begin{array}{c}\text { RT } \\
\text { modal. }\end{array}$ & A & Session & $\begin{array}{c}\text { number of } \\
\text { patients }\end{array}$ & $\begin{array}{c}\text { interval } \\
{[\text { months }]}\end{array}$ & $\begin{array}{c}\mathrm{CBF}_{\mathrm{A}} \\
\text { mean } \pm \text { std }[\mathrm{mL} / \mathrm{min} / 100 \mathrm{~g}]\end{array}$ & $\begin{array}{c}\Delta \mathrm{CBF} \\
\text { mean } \pm \text { std [\%] }\end{array}$ & $P$-value \\
\hline \multirow{2}{*}{ Photon } & Pre & Post-1 & 41 & $4.7 \pm 0.4$ & $53.4 \pm 9.7$ & $48.0 \pm 9.6$ & $-10.1 \pm 19.9$ & 0.002 \\
& Pre & Post-2 & 26 & $7.8 \pm 0.5$ & $53.4 \pm 10.7$ & $47.5 \pm 8.9$ & $-11.1 \pm 19.5$ & 0.008 \\
\hline \multirow{2}{*}{ Proton } & Pre & Post-1 & 15 & $4.8 \pm 0.5$ & $50.3 \pm 8.4$ & $45.8 \pm 8.6$ & $-9.1 \pm 21.6$ & 0.12 \\
& Pre & Post-2 & 11 & $8.0 \pm 0.6$ & $52.7 \pm 8.2$ & $48.0 \pm 11.1$ & $-8.8 \pm 19.7$ & 0.17 \\
\hline \hline \multirow{2}{*}{ 3DCRT } & Pre & Post-1 & 24 & $4.9 \pm 0.3$ & $53.0 \pm 8.8$ & $48.8 \pm 9.4$ & $-7.9 \pm 19.4$ & 0.061 \\
& Pre & Post-2 & 13 & $7.9 \pm 0.6$ & $51.8 \pm 8.8$ & $47.5 \pm 10.0$ & $-8.2 \pm 14.7$ & 0.07 \\
\hline \multirow{2}{*}{ IMRT } & Pre & Post-1 & 17 & $4.6 \pm 0.5$ & $53.9 \pm 11.2$ & $46.8 \pm 10.1$ & $-13.1 \pm 20.9$ & 0.01 \\
& Pre & Post-2 & 13 & $7.7 \pm 0.4$ & $55.0 \pm 12.4$ & $47.4 \pm 8.0$ & $-13.7 \pm 24.0$ & 0.055 \\
\hline
\end{tabular}

Perfusion changes are shown for photon- (top) and proton-therapy patients (bottom). On each line, the absolute $\mathrm{CBF}$, number of available patients, time between the sessions, the mean relative CBF difference between sessions, and the $P$-value of the paired t-test between the pre-therapeutic (Pre-) and one of the two post-therapeutic sessions (Post-1 and Post-2) are shown. The magnitudes of changes were similar for all sessions and RT modalities, however, the changes were significant only for the photon-therapy patients. Results are shown also for the two subgroups of the photon-therapy patients. While magnitude of changes is again similar, significant decrease is observed only on the first post-therapeutic session of the IMRT patients where the magnitude of decrease is higher than for the 3DCRT patient group. 
Table 5: Dose-dependent perfusion changes in the healthy hemisphere.

\begin{tabular}{ccccccc}
\hline Photon & Dose [Gy] & $0-10$ & $10-60$ & $10-20$ & $20-30$ & $30-60$ \\
\hline Pre & $\Delta$ CBF [\%] & -7.3 & -8.3 & -8.9 & -8.5 & -8.7 \\
$/$ & Region size [\%/n] & $26 \% / 36$ & $74 \% / 41$ & $28 \% / 40$ & $19 \% / 36$ & $26 \% / 41$ \\
Post-1 & P-value [0] & 0.057 & $0.009^{\star}$ & $0.006^{\star}$ & $0.017^{\star}$ & $0.007^{\star}$ \\
& P-value [LD] & - & 0.39 & 0.21 & 0.3 & 0.4 \\
\hline Pre & $\Delta$ CBF [\%] & -7.3 & -8.8 & -10.3 & -8.0 & -8.0 \\
$/$ & Region size [\%/n] & $27 \% / 21$ & $73 \% / 26$ & $29 \% / 26$ & $20 \% / 21$ & $24 \% / 25$ \\
Post-2 & P-value [0] & 0.15 & $0.029^{\star}$ & $0.007^{\star}$ & 0.09 & 0.061 \\
& P-value [LD] & - & 0.51 & 0.27 & 0.18 & 0.51 \\
\hline Proton & Dose [Gy] & $0-10$ & $10-60$ & $10-20$ & $20-30$ & $30-60$ \\
\hline Pre & $\Delta$ CBF [\%] & -7.0 & -9.5 & - & - & - \\
$/$ & Region size [\%/n] & $90 \% / 15$ & $10 \% / 8$ & $2 \% / 1$ & $2 \% / 3$ & $7 \% / 8$ \\
Post-1 & P-value [0] & 0.23 & 0.25 & - & - & - \\
& P-value [LD] & - & 0.75 & - & - & - \\
\hline Pre & $\Delta$ CBF [\%] & -8.3 & -7.4 & - & - & - \\
$/$ & Region size [\%/n] & $91 \% / 11$ & $9 \% / 7$ & $2 \% / 2$ & $1 \% / 3$ & $6 \% / 7$ \\
Post-2 & P-value [0] & 0.19 & 0.36 & - & - & - \\
& P-value [LD] & - & 0.43 & - & - & - \\
\hline
\end{tabular}

The mean relative perfusion changes are shown for different dose regions for photon- and proton-therapy patients. Note that effect size and $P$-values are not given for the finer division of the regions above 10 Gy for the protontherapy patients as the region size was too small to give meaningful results. The mean volume of the dose region as a percentage of the whole hemisphere, and the number of patients for which the region volume exceeded the threshold of $5 \mathrm{~cm}^{3}$ are shown as Region size. The $P$-values of the t-test indicate probability of no absolute change $[0]$ and no change relative to the low-dose (below $10 \mathrm{~Gy}$ ) region $[L D]$. Significant values are labeled by ${ }^{\star}$ for $P<0.05$, and by

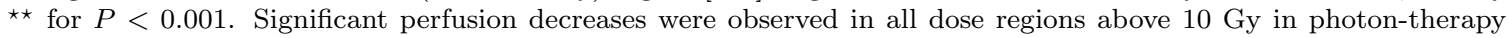
patients on the first post-therapeutic session. However, the results for regions above 10 Gy were not significantly different from the change in the region below $10 \mathrm{~Gy}$. Effect size was similar in the proton-therapy patients, though the differences were not statistically significant. 


\section{FIGURE LEGENDS}

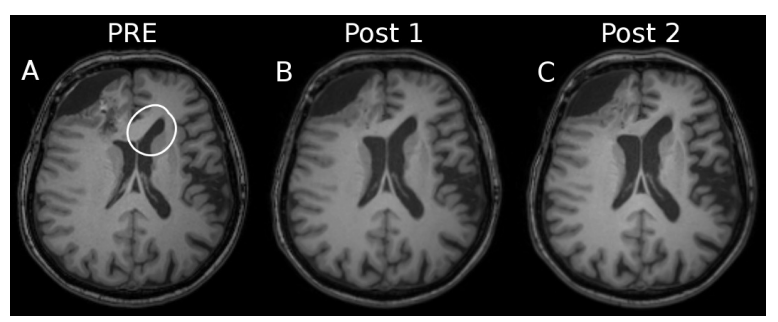

Figure 1: Progressive brain atrophy. T1w images of an IMRT photon-therapy patient are shown for pre- (A) and post-therapeutic $(\mathrm{B}, \mathrm{C})$ sessions. Brain atrophy manifests as ventrical and outer CSF space enlargement (see, for example, anterior portion of cella media). Tissue volume decrease on the first post-therapeutic session (B) compared to the pre-therapeutic baseline (A) was $1.8 \%$. Progression of the atrophy is observed on the second post-therapeutic session (C): $4.2 \%$ decrease compared to the pre-therapeutic baseline (A). 


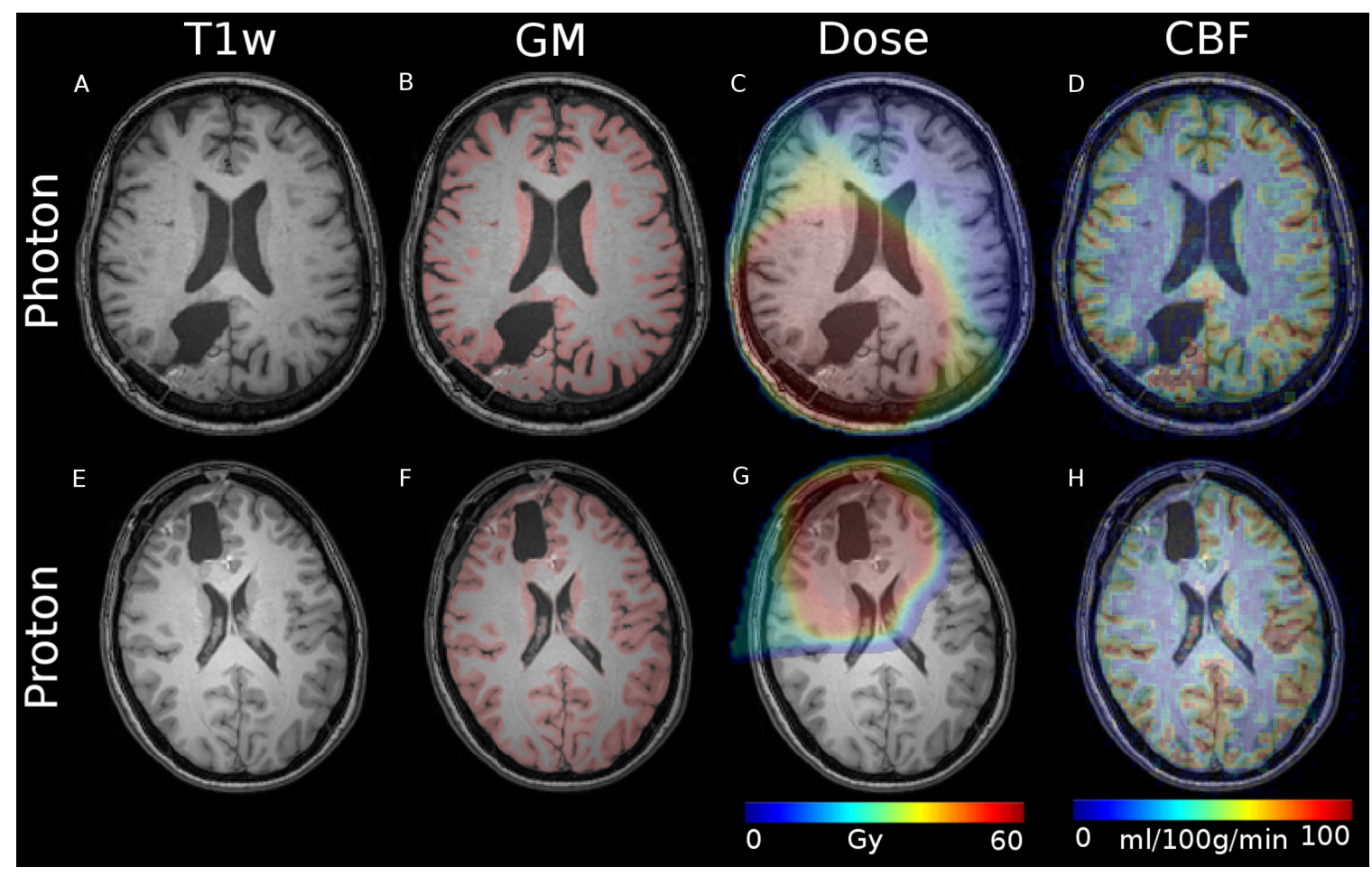

Figure 1: Images from representative patients are shown for 3DCRT photon (A-D) and proton (E-H) therapy. T1-weighted (T1w) images (A,E) were segmented to GM, WM and CSF in each voxel. The T1w are shown overlaid with the voxel-wise map of GM segmentation $(B, F)$, the calculated radiation-dose $(C, G)$, and the perfusion image $(\mathrm{D}, \mathrm{H})$. Note the difference in dose distribution between both radiotherapy $(\mathrm{RT})$ modalities and sparing of healthy tissue distal of the target volume achieved with proton therapy.

\begin{tabular}{cccc}
\hline & \multicolumn{2}{c}{ Photon } & Proton \\
Reason & 3DCRT & IMRT & \\
\hline WM changes [n] & 1 & 5 & 4 \\
Missing or motion [n] & 4 & 1 & 2 \\
Bilateral tumor [n] & 0 & 1 & 3 \\
\hline
\end{tabular}

Table 1: Exclusion from the volume analysis. Sessions with T1w images with visible changes in WM contrast in the healthy hemisphere compared to the pre-RCT session (edema, leukoencephalopathy, demyelinization), severe motion artifacts or missing $\mathrm{T} 1 \mathrm{w}$ data, and bilateral tumor presence, were excluded from the volumetric analysis. 

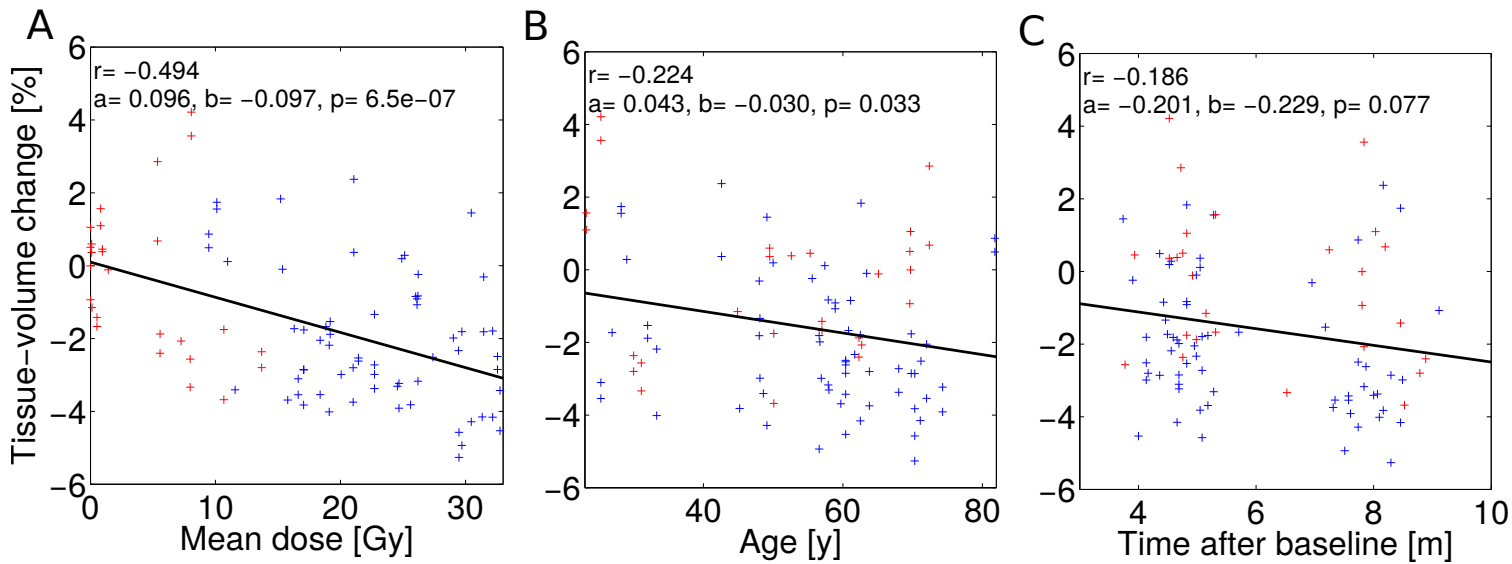

Figure 2: Univariate regression of global volume changes. The scatter plots show the dependence of tissuevolume changes on A) mean dose received in the hemisphere contralateral to the tumor, B) patient age, C) time after the baseline pre-therapeutic scan (blue: photon-therapy, red: proton-therapy) . The Pearson's correlation coefficient $(\mathrm{r})$ and the parameters and p-value of linear regression with $y=a+b \times x$ are given in each plot. While statistical significance is also reached for the age effect (B) only the dose effect (A) can be ascertained with very high statistical significance.

\begin{tabular}{cccc}
\hline & \multicolumn{2}{c}{ Photon } & Proton \\
Reason & 3DCRT & IMRT & \\
\hline Motion [n] & 0 & 2 & 1 \\
Labeling [n] & 0 & 1 & 0 \\
Bilateral tumor [n] & 0 & 1 & 2 \\
Missing ASL [n] & 1 & 0 & 4 \\
ATT artifacts [n] & 3 & 3 & 3 \\
\hline
\end{tabular}

Table 2: Exclusion from the perfusion analysis. ASL images with severe motion artifacts, failure of blood labeling, bilateral tumor presence, or sessions where ASL was not acquired for technical reasons were excluded from the perfusion analysis.

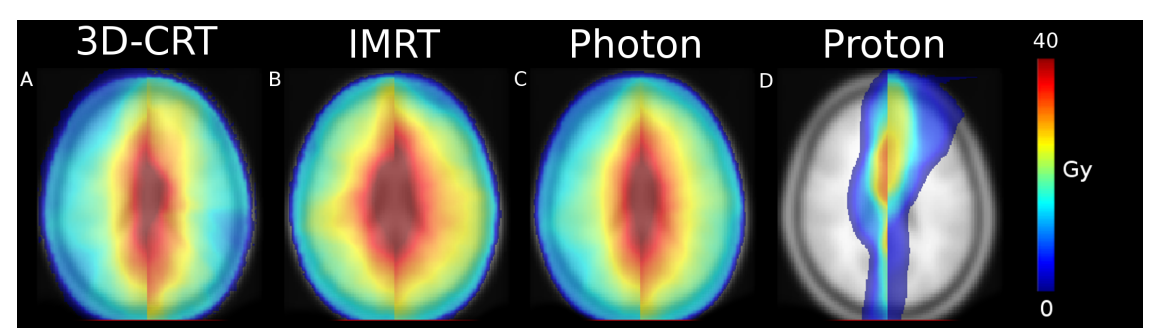

Figure 3: Mean dose in the healthy hemisphere for the two photon-therapy subgroups A) 3D conformal radiotherapy (3DCRT), B) intensity-modulated radiotherapy (IMRT), C) all photon- and D) all proton-therapy patients. Mean dose over the healthy hemisphere was calculated for each group and combined result is shown for patient with tumor in the left and in the right hemisphere which explains the discontinuity at the midline. 


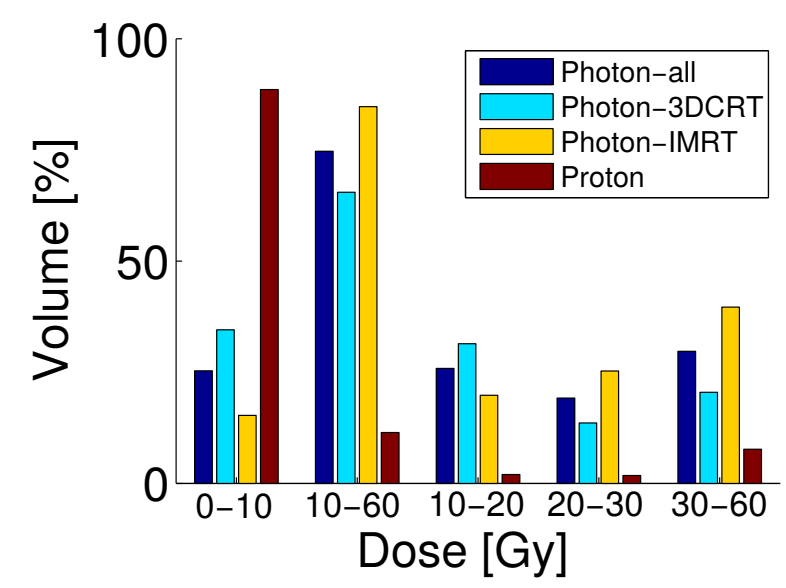

Figure 4: Dose distribution in the healthy hemisphere for the photon- and proton-therapy patients and the two photon-therapy subgroups. Mean relative volume for each isodose region is shown as a fraction of the whole-hemisphere volume. Note that both bins of 0-10 and 10-60 Gy, and bins of 0-10, 10-20, 20-30 and 30-60 Gy add up to $100 \%$. The proton-therapy clearly spares the healthy hemisphere more than all photon-therapies with only minimal volume receiving dose above 10 Gy. IMRT has slightly higher relative volume above 20 Gy than the 3DCRT planning which is both due to the inherent properties of the planning and slightly larger CTV in the IMRT group than in the 3DCRT group. 